\section{COVID-19 Pandemic: Public Trust and Guiding Ethics Where Ventilators Equal Lives}

Sir,

Severe COVID-19, presenting with acute respiratory distress syndrome (ARDS), for which mechanical ventilation is the mainstay treatment. Current available data is insufficient to draw safe conclusion but suffice to say that it would be worse than ARDS due to other causes. It is not clear in the absence of definitive data to know about the prognosis of mechanical ventilation. This letter addresses the guiding ethical principles involved in ventilating the COVID-19 patients. Who gets the ventilator? Doctors are challenged to decide now who gets care if COVID-19 overwhelms emergency department and intensive care units. As pandemic intensifies and a crisis for medicines, testing agents, ventilators, and personal protection equipment, we may end up in a situation where we have to make tough ethical decisions. Criterion has been made on the principles of utility and efficiency to promote the actions for greater good. Another ethical principle, equity require consideration. ${ }^{1}$ Public resources are being used to address a public health hazard; hence, public stakeholders are essential in decision making for allocating beds and ventilators.

COVID-19 with ARDS, in which many intensivists tried prone positioning with success, may decrease the need of intubation and may avoid ventilator demand in the crisis. However, many hospital settings are not aware or equipped for it. $^{2}$ Despite receiving supplemental oxygen or fatigue due to severe inflammation in COVID-19 patients, many patients require bag-mask ventilation as a temporary measure until definitive measure of intubation is performed. Given the option for ventilation, identifying both ethical and pragmatic principles, is the recognition where public trust can be gained. It requires reinforcement from public health experts to emphasise on transparency.

COVID-19 brought this to light how fragile the supply chain is. With shortage of ventilators, healthcare workers are having encounters such as, should they withdraw ventilators from some patients and use them for other patients, who have a chance of survival. What criteria should be used to allocate the ventilators will be context-dependent. ${ }^{2}$

COVID-19 poses another scenario: patients who do not receive a ventilator could benefit and living for many more years if they got short-term mechanical ventilation. This pandemic called for the rationing and reallocation of ventilators in a fashion that aims for saving maximum number of lives. Medications included sedatives, anesthetics, analgesia, muscle relaxants; and their shortage raise a challenge to healthcare workers to place these patients on ventilators. Multiple criteria should be used into a single tool to prioritise patient care in a local setting as no single criteria fulfill morally relevant values. ${ }^{3}$ With the loaming threat, we need to find a way for more fair allocation of these scarce resources.

Physicians are grappled by grim moral choices where it was thought that only COVID-19 patients, who got decent chance of survival and potential life years, will get access to beds and ventilators. These questions require calm and rationalisation. It is quite evident that intensive care units will collapse under the dramatic spike of COVID-19 cases, in particular, when no one has a firm grasp on how community spread is occurring. ${ }^{4} \mathrm{Health}-$ care workers are challenged by the allocation of ventilators and felt it will not be enough if we do not bring down the curve. We are finding quite dysfunctional emergency departments these days with concerned patients and family members. Hospital boards and public discussions should be on the roll until our physicians get exhausted and overworked. It is no longer an academic exercise now.

Rationing of ventilators with other scare supplies will depend on contextual factors as they change. Guiding ethical principles of efficiency and utility should go with equity. COVID-19 is a public health emergency. ${ }^{4}$ The geopolitical shockwaves are emitting a lesson, which should be learned quite sometime back.

But that is what globalisation offers.

\section{CONFLICT OF INTEREST:}

Authors declared no conflict of interest.

\section{AUTHORS' CONTRIBUTION:}

SS, MAB: Substantial contributions to the design of the work; drafting the work and revising it critically for important intellectual content; approved the final version to be published.

\section{REFERENCES}

1. Rosenbaum L. Facing Covid-19 in Italy - ethics, logistics, and therapeutics on the epidemic's front line. $N$ Engl J Med 2020; 382(20):1873-75.

2. Wang D, Hu B, Hu C, Zhu F, Liu X, Zha J, et al. Clinical characteristics of 138 hospitalised patients with 2019 novel coronavirus-infected pneumonia in Wuhan, China. JAMA 2020; 323(11):1061-9.

3. Lew TWK, Kwek TK, Tai D, Earnest A, Loo S, Singh K, et al. Acute respiratory distress syndrome in critically ill patients with severe acute respiratory syndrome. JAMA 2003; 290(3):374-80.

4. Hongliang W, Sicong W, Kaijiang Y. COVID-19 infection epidemic: The medical management strategies in heilongjiang province, china. Crit Care 2020; 24(1): 107.

Sadaf Sheikh ${ }^{1}$ and Muhammad Akbar Baig ${ }^{2}$

${ }^{1}$ Department of Emergency Medicine, Sultan Qaboos University Hospital, Muscat, Oman

${ }^{2}$ Department of Emergency Medicine, The Aga Khan University Hospital, Karachi, Pakistan 
Correspondence to: Dr. Sadaf Sheikh, Department of Emergency Medicine, Sultan Qaboos University Hospital, Muscat, Oman

E-mail: sheikh.sadaf@gmail.com
(1).....

Received: April 04, 2020; Revised: May 30, 2020;

Accepted: June 24, 2020

DOI: https://doi.org/10.29271/jcpsp.2020.JCPSPCR.CR74

$\bullet \bullet \bullet \bullet \bullet \bullet \bullet \bullet \bullet$ 